\title{
STUDIES ON THE COURSE OF VASOMOTOR FIBERS AS \\ MEASURED BY THERMIC CHANGES IN THE FEET \\ AFTER ARTERIAL LIGATION AND SECTION OF THE SPINAL CORD AT VARIOUS LEVELS
}

\author{
By ASHLEY W. OUGHTERSON, SAMUEL C. HARVEY, \\ AND HELEN G. RICHTER \\ (From the Department of Surgery, Yale University School of Medicine, New Haven)
}

(Received for publication June 4, 1932)

In a previous paper (7) it was shown that in dogs under amytal anesthesia, ligation of both femoral arteries proximal to the profunda branch caused a decrease in the temperature of the feet which was interpreted as indicating a diminution of blood flow. The temperature of the feet remained at this lower level for several hours following which there was a spontaneous rapid rise indicating the development of collateral circulation. The rapidity of this response was taken as evidence suggesting that the reestablishment of former conditions by collateral circulation is a vasomotor phenomenon. It was found furthermore that decrease in the temperature of the feet following ligation of the femoral artery as above, could be prevented by removal of the tributary sympathetic ganglia, or if the temperature had already decreased to the level of that of the room, removal of the sympathetic ganglia immediately induced a rise in the feet to the previous level. This suggested that the rapid spontaneous return of the circulation following ligation of the femoral artery was a vasomotor phenomenon controlled by means of the sympathetic nervous system.

In another paper (3) experiments were reported in which the same method was used. Following transection of the spinal cord at the level of the first lumbar vertebra, no increase in the temperature of the limb was observed. Gaskell (2), Bayliss and Bradford (1) and Langley (4, 5, 6 ) have stated that in an animal with seven lumbar vertebrae the connector fibers to the sympathetic ganglia supplying the posterior extremity are contained in the 11th, 12th and 13th thoracic and the 1st, 2nd and 3rd lumbar spinal roots. Since the results of the above (3) experiments did not coincide with their findings it seemed desirable to conduct further experiments to determine the effect of transection of the spinal cord at various levels.

\section{METHOD}

As in the previous experiments, dogs were given sodium amytal (Lilly) intraperitoneally and the temperatures of the room, rectum and hind feet 
recorded simultaneously by means of thermocouples attached to a Leeds and Northrup resistance thermometer. The experimental procedure was, however, modified in some respects. Unpublished experiments show that there is a correlation between body temperature and the temperature reaction of the extremities. The body temperature was therefore maintained at normal by use of blankets and by means of electric heating pads when necessary. Inasmuch as many of the experiments extended over a period of 24 hours, the preliminary intra-abdominal ligations of the arteries were accomplished using sterile technique to minimize general temperature reactions. During the course of the experiments it was found that transection of the spinal cord at a given level did not always result in the same changes in temperature in the extremity but that the effects varied considerably in the different animals, apparently under the same conditions. This was most frequently found when the level of transection was below the first lumbar nerve root.

Other investigators (Langley, (4) Gaskell (2), Bayliss and Bradford (1)) have demonstrated that vasomotor fibers supplying the lower limbs leave the spinal cord from the eleventh thoracic to the third lumbar nerves. It was therefore apparent that transection of the spinal cord at the lower levels would interrupt fewer vasomotor fibers than at the higher. It would seem that failure of the temperature to rise or of the blood flow to increase did not necessarily mean that no vasomotor fibers had been interrupted but rather that a sufficient number had not been cut to result in increased blood flow. It also seemed probable that if the femoral artery were ligated above the profunda branch, it would be necessary to interrupt more fibers in order to result in a rise of temperature of the foot than if the ligation were done below the profunda branch. Hence in some of these experiments the ligation was carried out in the groin below the profunda branch. The dogs were taken from stock without preliminary dietary standardization. Preference was given to young animals although some of them were obviously old.

Following the fall in temperature of the foot after ligation laminectomy was performed at the desired level, the dura was opened, and the spinal cord transected. When the cord was exposed the dentate ligaments at the desired level were cut and the cord gently lifted with a blunt hook and cut with scissors. There was usually very little bleeding which could readily be controlled by cotton pledgets or by placing a piece of muscle between the ends of the transected cord. Following this the temperature changes in the hind feet were noted as shown in the charts.

\section{EXPERIMENTAL DATA}

In this group of experiments in which the spinal cord was transected at various levels, twenty-one dogs were used. Two dogs failed to exhibit vasodilatation even after removal of the sympathetic chain and section of the sciatic nerve. One of these (Experiment 146, Protocols) was an old animal in poor condition; during amytal anesthesia this animal developed a marked bronchorrhea. One animal (Experiment 15) also failed to react both on transection of the cord and likewise following removal of the lumbar sympathetic chain. The femoral arteries of this animal were ligated at the bifurcation resulting in a.slight fall only in the temperature of the feet. The temperature of the room was $80^{\circ} \mathrm{F}$. Inasmuch as three hours following ligation, the temperature of the feet 
was still $94^{\circ} \mathrm{F}$. it is probable that vasodilatation was already present and that this explains the absence of change following operation. Experiment 30 was also inconclusive. A fall in temperature following ligation of the arteries took place, but no rise occurred after transections at $\mathrm{L} 4{ }^{1}$ and $\mathrm{L} 2$. Following transection of the cord at $\mathrm{T} 10^{1}$ an increase of $7^{\circ} \mathrm{F}$. occurred in the temperature of the feet, but was not sustained. At the end of two hours the feet were again at room temperature. Following this, removal of the sympathetic ganglia did not result in increase of the temperature of the feet.

All of the other eighteen dogs reacted well either to transection of the cord or removal of the sympathetic ganglia. The response obtained in each of the experiments will be found in the protocols. The terms "Immediate" and "Delayed" refer to the time when increase in temperature occurred and the terms "Abrupt" and "Gradual," to the type of curve. It is to be noted that vasodilatation always resulted when the cord was transected above the level of the second lumbar nerve root. However, in Experiment 30 there was none at L2, in Experiment 27 at L3, in Experiments 30 and 31 at L4 and in Experiment 139 at L5. In all of these experiments except 139 the ligation was at the bifurcation. In Experiment 139 the right side was ligated at the bifurcation and the left side below Poupart's ligament. When it was performed below Poupart's ligament (i.e. below the profunda branch) immediate vasodilatation took place at the lower levels, as in Experiments 135, 136 and 140.

\section{DISCUSSION}

The experiments reported in this paper seem to indicate that vasomotor pathways can be traced satisfactorily by this method. The results are, however, not always consistent as there are several variable factors which may lead to erroneous interpretation. It may be well therefore to review some of the variables in order that the results may be more easily interpreted.

The reaction of dogs to amytal anesthesia is not constant and its effect on the sympathetic nervous system is not well understood. These experiments demonstrate that some dogs under amytal anesthesia are no longer able to maintain normal temperature in a room of $70^{\circ} \mathrm{F}$. Certain dogs do so, however, for long periods. While long haired dogs perform this function better than short haired dogs, this is not always the case. The important result is that dogs under amytal anesthesia conserve their body heat by vasoconstriction or vasodilatation, depending upon environmental temperature and the peculiarities of the individual dog. Vasoconstriction as a result of low body temperature may be sufficient to counteract the effect of interrupting a few vasoconstrictor

${ }^{1} \mathrm{~L} 4$ represents fourth lumbar nerve root, T10 represents tenth thoracic nerve root. In each instance the transection was carried out immediately caudad to the root named. 
fibers. When the body temperature is normal, interruption of the same number of vasoconstrictor fibers is made manifest by a rise in the temperature of the limb in question. In spite of the obvious effect of amytal anesthesia on the conservation of body heat, relatively small changes in the reaction of the sympathetic nervous system may, nevertheless, be demonstrated providing body heat is artificially maintained at a normal level. There is also considerable variation in the reaction of the vasomotor system of various dogs. Certain animals under standard conditions respond with marked vasoconstriction while others apparently under the same conditions exhibit vasodilatation. In addition to variation in physiological response there are also anatomical variations. It was found that when the femoral artery was ligated above the profunda branch, the collateral circulation resulting from interruption of the sympathetic fibers was by way of the median sacral artery. When the median sacral artery was ligated as well as the femoral, no rise of temperature could be produced in that limb. Inasmuch as there is considerable variation in the portion of the limb supplied by these arteries, the experiments cannot be considered identical in the anatomical sense.

Previous studies (Langley (4) (5) (6), Bayliss and Bradford (1), Gaskell (2)) have shown that vasomotor fibers leave the spinal cord from the level of the second thoracic to the third lumbar spinal nerve roots. Various experimental methods were used in determining these levels. In cats, Langley (5), observed the secretion of sweat in the foot pad as well as the color of the pad when the individual nerve roots were cut and stimulated. Gaskell carried out extensive anatomical and histological studies on the nerve roots and rami communicantes of dogs which were essentially in agreement with the findings of Langley. Bayliss and Bradford using a plethysmograph recorded the changes in volume of the limb following stimulation of the individual nerve roots and also agreed with the findings of Gaskell and Langley.

The experiments recorded in the protocols demonstrate the quantitative aspects of vasodilatation depending on the number of fibers interrupted and the level of ligation as well as on the other factors enumerated above. Transection of the spinal cord as low as the sixth lumbar nerve root resulted in vasodilatation of the vessels of the extremity providing the means for recording dilatation did not require the interruption of a large number of fibers. In other words, a sufficient number of vasoconstrictor fibers leave the spinal cord below the level of the sixth lumbar nerve root to result in vasodilatation when these fibers are interrupted. Transection of the spinal cord interrupts of course all of the fibers leaving the cord below this level. It is probable that interruption or stimulation of a single nerve root containing a few fibers would not result therefore in sufficient vasodilatation to be recorded by the means used. The response obtained by ligation at various levels indicates the 


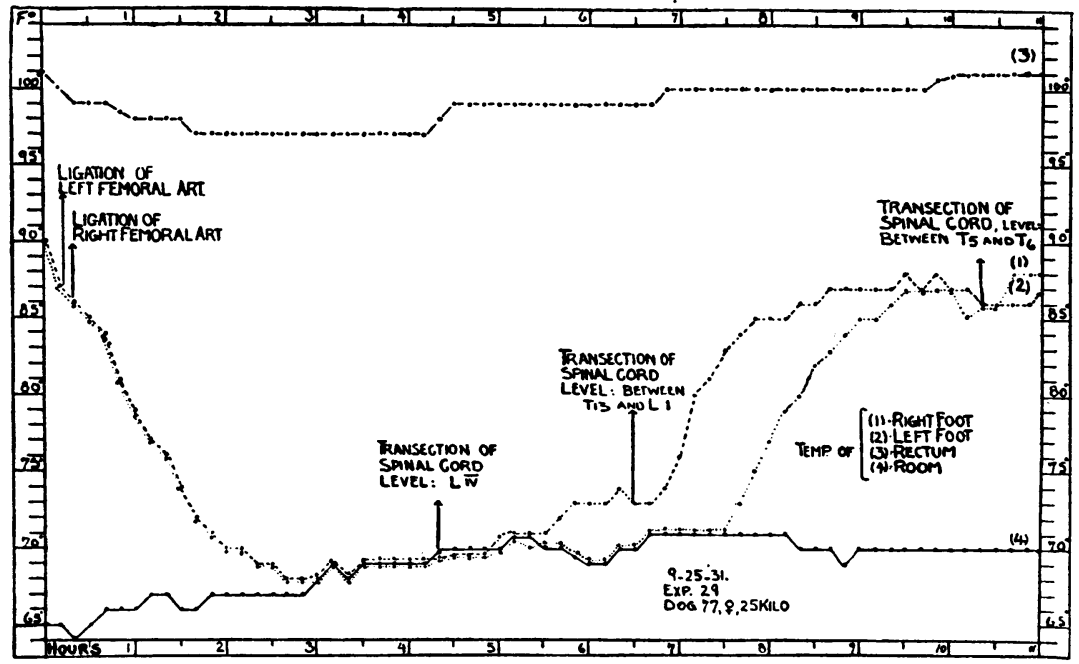

Fig. I. EXPERIMENT 29

The femoral arteries were ligated just below the bifurcation of the aorta. Curves show a slight rise of temperature of the left foot after transection of the spinal cord below L4 nerve roots. Transection below T13 resulted in an immediate, abrupt, sustained rise on the left, and a delayed, abrupt, sustained rise on the right.

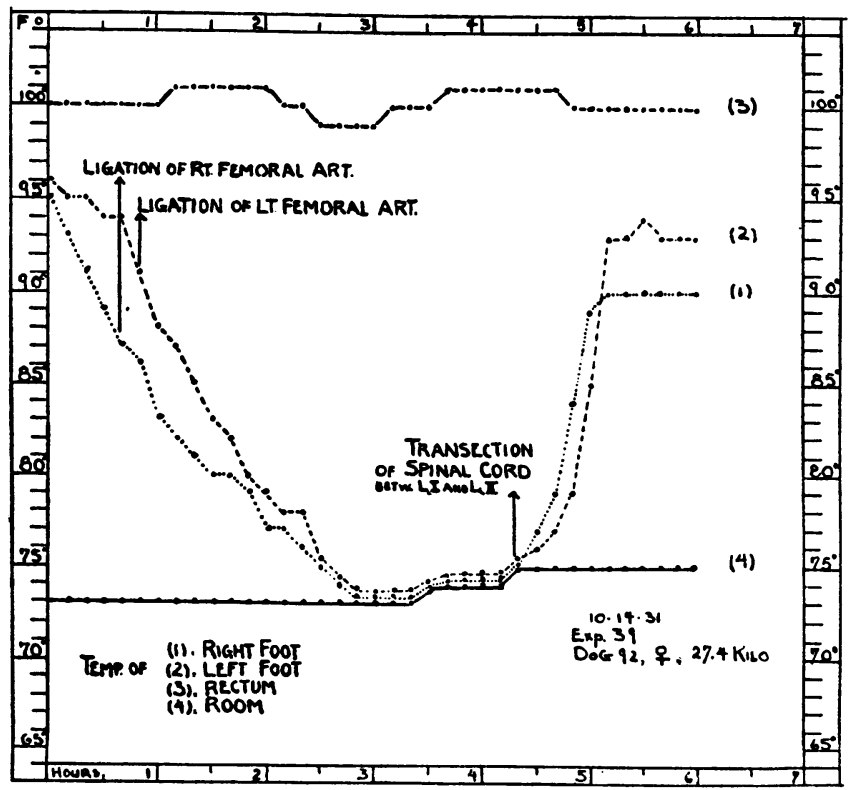

Fig. II. EXPERIMENT 39

The femoral arteries were ligated just below the bifurcation of the aorta. Transection of the spinal cord below $\mathrm{L} 1$ nerve roots resulted in an immediate abrupt sustained rise of the temperature of both feet. 


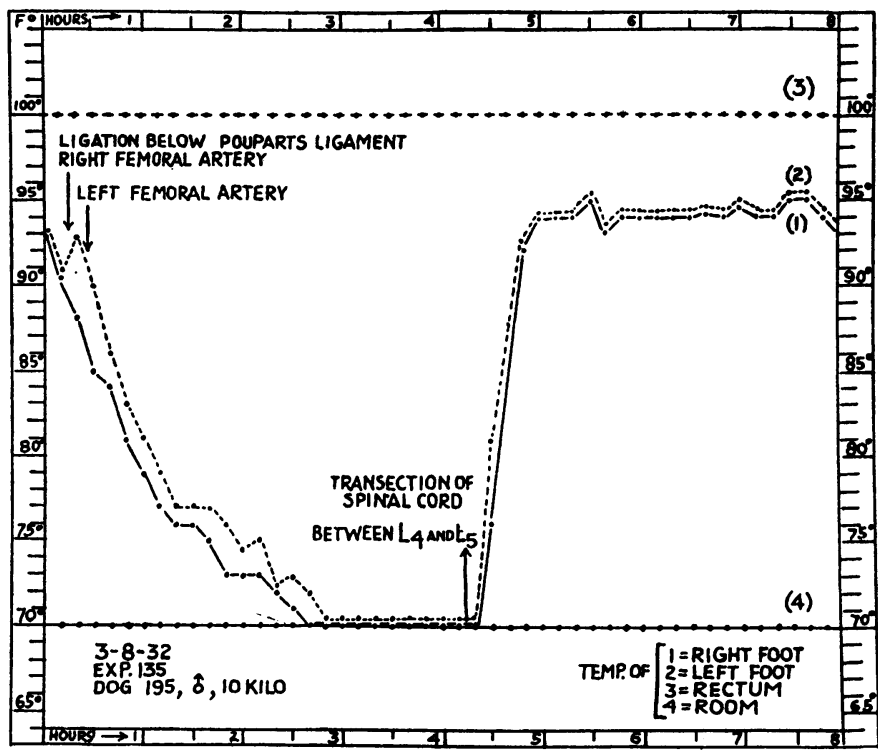

Fig. III. EXPERIMENT 135

The femoral arteries were ligated below Poupart's ligament (below profunda branch). Transection of the spinal cord below L4 nerve roots resulted in an immediate abrupt sustained rise of the temperature of both feet.

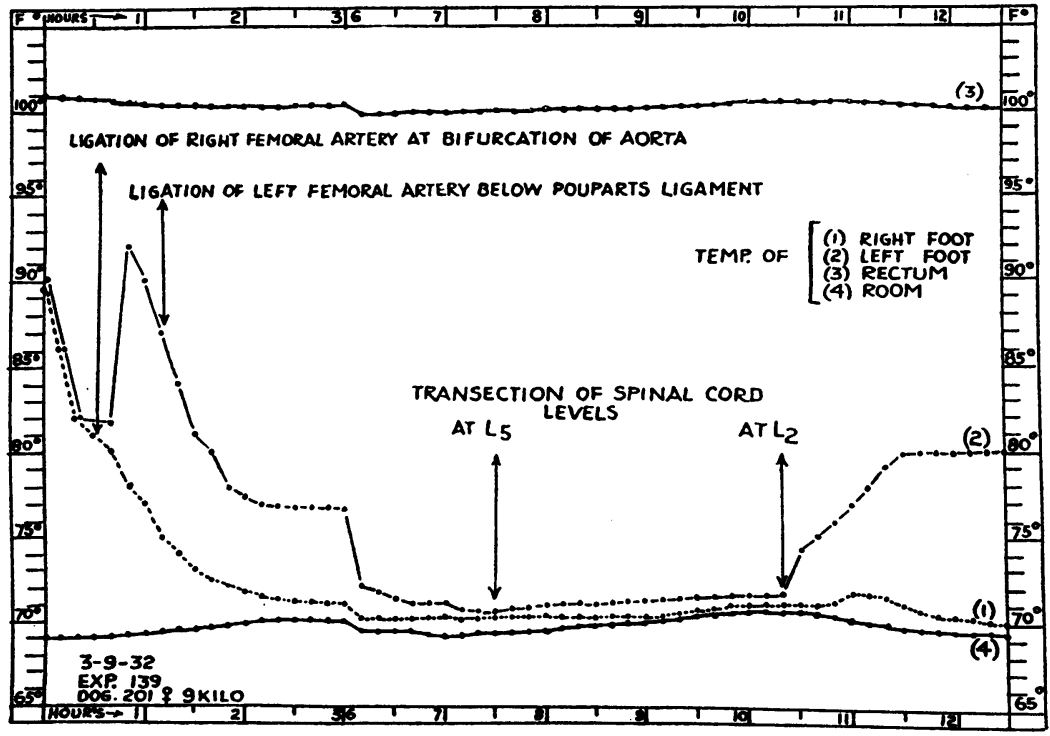

Fig. IV. EXPERIMENT 139

The curves show the difference in response to cord transection when the femoral artery is ligated just below the aorta on the right and below Poupart's ligament on the left. 
quantitative aspect of the methods used for recording vasodilatation, the limits of which have as yet not been fully determined. Further work on the course of vasomotor fibers is being carried on in this laboratory.

\section{SUMMARY}

1. The vasomotor effects of spinal cord transection at various levels have been studied in the dog following the ligation of the femoral artery at various levels.

2. The temperature changes in the hind feet following ligation of the arteries and transection of the spinal cord are dependent on both the level of transection of the cord (number of vasomotor fibers interrupted) and the level of ligation of the artery as well as on other factors which are discussed.

3. If the femoral artery is ligated just below the bifurcation of the aorta, transection of the spinal cord above the level at which the vasomotor fibers are given off to the hind limb is followed by immediate full vasodilatation. Under the same conditions transection of the spinal cord below the level of the first lumbar nerve root does not always bring about a vasodilatation of sufficient degree to be recorded by the method used in these experiments. This explains the failure to obtain vasodilatation as previously reported (3). Partial or incomplete vasodilatation may however, be observed in some animals.

4. If the femoral artery is ligated below the profunda branch (below Poupart's ligament) transection of the spinal cord at lower levels will cause immediate full vasodilatation.

5. Using this method it was found that there is a sufficient number of vasomotor fibers leaving the spinal cord below the level of the sixth lumbar nerve root to bring about immediate vasodilatation when they are interrupted.

\section{CONCLUSIONS}

These experiments suggest that, if the method of measuring vasodilatation is capable of recording the changes resulting from division of a small number of fibers, vasomotor fibers are found to leave the spinal cord at lower levels than have previously been described.

\section{PROTOCOLS}

Summary of twenty-one experiments in which the spinal cord was transected

Experiment 15. Room temperature $80^{\circ} \mathrm{F}$.; femoral arteries ligated below bifurcation of the aorta; spinal cord transected at level of T8. Result: No response after transection of the cord; no response after removal of the sympathetic ganglia. Vasodilatation present at time of transection, hence no further response.

Experiment 16. Room temperature $80^{\circ} \mathrm{F}$.; femoral arteries ligated below bifurcation of the aorta; spinal cord transected at level of T5. Result: There was an immediate abrupt sustained rise of $14^{\circ} \mathrm{F}$. in both feet. 
Experiment 19. Room temperature $82^{\circ} \mathrm{F}$; femoral arteries ligated below bifurcation of the aorta; spinal cord transected at level of T5. Result: There was an immediate abrupt sustained rise of $10^{\circ} \mathrm{F}$. in both feet.

Experiment 24. Room temperature $82^{\circ} \mathrm{F}$; femoral arteries ligated below bifurcation of the aorta; spinal cord transected at level of L1. Result: There was an immediate gradual sustained rise of $14^{\circ} \mathrm{F}$. in the right foot, and an immediate rise in the left foot from $94^{\circ}$ to $100^{\circ} \mathrm{F}$. The temperature of the left foot had not decreased with that of the right.

Experiment 25. Room temperature $80^{\circ} \mathrm{F}$; femoral arteries ligated below bifurcation of the aorta; spinal cord transected at level of T12. Result: A slightly delayed but abrupt and sustained rise of $8^{\circ} \mathrm{F}$. in both feet.

Experiment 26. Room temperature $77^{\circ} \mathrm{F}$; femoral arteries ligated below bifurcation of the aorta; spinal cord transected at level of L2. Result: An immediate abrupt sustained rise of $12^{\circ} \mathrm{F}$. in both feet. There was also a slight additional rise after sympathectomy.

Experiment 27. Room temperature $77^{\circ} \mathrm{F}$.; femoral arteries ligated below bifurcation of the aorta; spinal cord transected at the level of L3. Result: There was no change in temperature after the transection but an immediate rise of $20^{\circ} \mathrm{F}$. after sympathectomy.

Experiment 28. Room temperature $72^{\circ} \mathrm{F}$; femoral arteries ligated below bifurcation of the aorta; spinal cord transected at the level of L2. Result: An immediate gradual sustained rise of $20^{\circ} \mathrm{F}$. in the right foot, a delayed gradual slight rise in the left foot, and a rise of only $6^{\circ} \mathrm{F}$. in the left foot after sympathectomy. Following ligation, the temperature of the left foot fell much more abruptly to room temperature.

Experiment 29. Room temperature $70^{\circ} \mathrm{F}$; femoral arteries ligated below bifurcation of the aorta. With transection of the spinal cord at L4 there was an immediate gradual rise of $4^{\circ} \mathrm{F}$. in the left foot only; with transection at L2 there was an immediate abrupt sustained rise of $14^{\circ} \mathrm{F}$. in the left foot and a delayed gradual sustained rise of $16^{\circ} \mathrm{F}$. in the right foot. With transection at T6 there was an immediate rise of $2^{\circ} \mathrm{F}$. in both feet.

Experiment 30 . Room temperature $70^{\circ} \mathrm{F}$; f femoral arteries ligated below bifurcation of the aorta. Transecting the spinal cord at the level of $\mathrm{L} 4$ elicited no response in either foot, as was also the case with transection at L2. Upon transecting the cord at the level of T10 there was an immediate gradual rise of $7^{\circ} \mathrm{F}$. in both feet lasting 2 hours, but again on transecting at the level of T5 there was no response. There was no response after removal of the sympathetic ganglia.

Experiment 31. Room temperature $70^{\circ} \mathrm{F}$; femoral arteries ligated below bifurcation of the aorta. Transection of the spinal cord at the level of L4 gave no response; upon transection at the level of L2 there was an immediate gradual sustained rise of $10^{\circ} \mathrm{F}$. in the left foot and $3^{\circ} \mathrm{F}$. in the right foot; transection at the level of $\mathrm{T} 10$ was followed by an immediate abrupt further rise of $16^{\circ} \mathrm{F}$. in the left foot and $4^{\circ} \mathrm{F}$. in the right. Transection at the level of T7 gave no response. The temperature of the right foot had been $8^{\circ} \mathrm{F}$. below that of the left before ligation and the fall of the temperature of the right foot to room temperature after ligation of the femoral arteries was much more abrupt.

Experiment 39. Room temperature $73^{\circ} \mathrm{F}$; f femoral arteries ligated below bifurcation of the aorta. The spinal cord was transected at the level of L1. There was an immediate abrupt sustained rise of $21^{\circ} \mathrm{F}$. in the left foot and $16^{\circ} \mathrm{F}$. in the right foot. Upon transection of the cord at the level of L1 on the left the root was cut, but on the right the root was intact. 
Experiment 41. Room temperature $74^{\circ} \mathrm{F}$.; femoral arteries ligated below bifurcation of the aorta; spinal cord transected at the level of L1. Result: Immediate abrupt sustained rise of $22^{\circ} \mathrm{F}$. in both feet. The rectal temperature of this animal was $103^{\circ} \mathrm{F}$. at the termination of the experiment.

Experiment 71 . Room temperature $72^{\circ} \mathrm{F}$; f femoral arteries ligated below Poupart's ligament and below profunda artery; spinal cord transected hemilaterally at the level of T9 on the left and at T10 on the right. Result: Immediate abrupt sustained hemilateral rise of $18^{\circ} \mathrm{F}$. in the left foot, and a delayed abrupt sustained hemilateral rise of $18^{\circ} \mathrm{F}$. in the right foot.

Experiment 72. Room temperature $70^{\circ} \mathrm{F}$.; femoral arteries ligated below Poupart's ligament and below profunda artery. An anterior quadrilateral section was done at the level of T10 on the left followed by a similar procedure on the right. Result: There was an immediate abrupt sustained rise of $18^{\circ} \mathrm{F}$. in the left foot and a delayed gradual sustained rise of $4^{\circ} \mathrm{F}$. in the right foot. A deeper anterior quadrilateral section was then carried out at the level of T12 on the right which was followed by an immediate abrupt further rise of $10^{\circ} \mathrm{F}$. in the right foot.

Experiment 135 . Room temperature $72^{\circ} \mathrm{F}$; femoral arteries ligated below Poupart's ligament and below profunda artery; spinal cord transected at the level of L5. Result: Immediate abrupt sustained rise of $22^{\circ} \mathrm{F}$. in both feet.

Experiment 136: Room temperature $70^{\circ} \mathrm{F}$; femoral arteries ligated below Poupart's ligament on both sides, and the profunda artery was also ligated on the left; spinal cord transected at level of L5. Result: Immediate abrupt sustained rise of $18^{\circ} \mathrm{F}$. in right foot, and immediate gradual sustained rise of $12^{\circ} \mathrm{F}$. in the left foot. The ligation of the profunda artery occasioned a slower rise to a lower level on the left.

Experiment 139. Room temperature $70^{\circ} \mathrm{F}$.; femoral artery on the right ligated at the bifurcation of the aorta, and on the left below Poupart's ligament; spinal cord transected at the level of L5, with no response on either side; spinal cord transected at the level of L2 with an immediate gradual not sustained rise of $10^{\circ} \mathrm{F}$. in the left foot and $3^{\circ} \mathrm{F}$. in the right foot.

Experiment 140 . Room temperature $67^{\circ} \mathrm{F}$.; femoral arteries ligated below Poupart's ligament; spinal cord transected at the level of L6 with a resultant immediate abrupt unsustained $\left(1 \frac{1}{2}\right.$ hours) rise of $12^{\circ} \mathrm{F}$. in both feet. Later transection of the cord at the level of L3 brought no response in either foot. There was a rise of temperature after section of the sciatic nerve of $16^{\circ} \mathrm{F}$. on the right and $6^{\circ} \mathrm{F}$. on the left. This dog required a larger dose of amytal than usual.

Experiment 146 . Room temperature $68^{\circ} \mathrm{F}$; f femoral arteries ligated below Poupart's ligament. There was no response to transection of the spinal cord at the level of L6, nor L3. Neither was there any response in the sciatic section (bilaterally). This was an old dog with much bronchorrhea, and a deep anesthesia was obtained.

\section{BIBLIOGRAPHY}

1. Bayliss, W. M. and Bradford, J. R., J. Physiol., 1894, xvi, 10. The innervation of the vessels of the limbs.

2. Gaskell, W. H., The involuntary nervous system. Longmans Green \& Co., London, 1916.

3. Harvey, Samuel C. and Halpert, Béla, J. Clin. Invest., 1931, x, 431. Studies on collateral circulation. II. Thermic changes after arterial ligation, section of spinal cord or posterior roots and ganglionectomy. 
4. Langley, J. N., The autonomic nervous system. Part I. W. Heffer and Sons, Cambridge, 1921.

5. Langley, J. N., J. Physiol., 1891, xii, 347. On the course and connection of the secretory fibers supplying the sweat glands of the feet of the cat.

6. Langley, J. N., J. Physiol., 1894, xv, 176. The arrangement of the sympathetic nervous system based chiefly on observations upon pilomotor nerves.

7. Mulvihill, Daniel A. and Harvey, Samuel C., J. Clin. Invest., 1931, x, 423. Studies on collateral circulation. I. Thermic changes after arterial ligation and ganglionectomy. 